JOURNAL REVIEW

\section{Oral contraceptive use and cancer: final report from the Oxford-Family Planning Association contraceptive study}

Vessey M, Yeates D. Contraception 2013;88: 678-683.

The Oxford-Family Planning Association (Oxford-FPA) contraceptive study has been providing evidence of contraceptive risks and benefits for nearly 40 years and will be a study with which most readers will be acquainted. This is the final report on cancer incidence from this study. Over 17000 subjects were initially recruited in the UK between 1968 and 1974 and were chosen mainly to look at oral contraceptive (OC) use. At the time, most OCs contained $50 \mu \mathrm{g}$ estrogen. The subjects at recruitment were made up of three groups: those using OCs, those using a diaphragm and those using an intrauterine device. Follow up continued until the end of 2010.

The results are in keeping with previously reported evidence. Reassuringly, breast cancer incidence was not affected by OC use [relative risk (RR) 1.0; 95\% confidence interval (CI) $0.9-1.1$ ]. This was the most common cancer in the study and the finding was based on 1087 women having developed the disease. With regard to gynaecological cancers, the numbers affected are fewer (61, 124 and 143 for cervix, uterine body and ovary, respectively). There was a significant increase in cervical cancer risk (RR 3.4; 95\% CI 1.6-8.9) with OC use. This mirrors larger epidemiological studies. ${ }^{1}$ Smoking habit and other confounding factors were taken into account, so the mechanisms behind this finding remain unexplained. Is this an epidemiological finding or is there true biological causation? This point remains unclear. No data are presented on incidence by histological subtype; however, the numbers of cervical adenocarcinomas are not likely to be sufficient to reveal any meaningful association with one histiotype or another. Uterine body (endometrial) cancer and ovarian cancer appear to benefit from a protective effect of OC use with RRs of 0.5 (95\% CI $0.3-0.7$ ) and 0.5 (95\% CI 0.4-0.7), respectively. Again these results have been found in other studies. What is less commonly described is the persistence of cancer protection for around 30 years for both endometrial and ovarian cancers. As the gynaecological oncology community now views the ovarian cancers as heterogeneous conditions, it is perhaps flawed to be examining cases of 'ovarian cancer' as one entity, as there are undoubtedly different oncogenic mechanisms involved in the various types.

Whilst the prospective and lengthy extent of this study are admirable, it can be argued that the present report adds little to the current state of knowledge of cancer risk and OC use in the 1970s, 1980s and 1990s.

Reviewed by K Scott Fegan

Consultant Gynaecological Oncologist, Royal Infirmary of Edinburgh, Edinburgh, UK; scott.fegan@nhs.net

Competing interests None.

\section{Provenance and peer review}

Commissioned; internally peer reviewed.

J Fam Plann Reprod Health Care 2014;40:112.

doi:10.1136/jprhc-2014-100909

\section{REFERENCE}

1 International Collaboration of Epidemiological Studies of Cervical Cancer, Appleby P, Beral V, Berrington de González A, et al. Cervical cancer and hormonal contraceptives: collaborative reanalysis of individual data for 16,573 women with cervical cancer and 35,509 women without cervical cancer from 24 epidemiological studies. Lancet 2007;370:1609-1621. 